\title{
Molecular Dynamics Simulations of CdTe / CdS Heteroepitaxy - Effect of Substrate Orientation
}

\author{
Jose J. Chavez ${ }^{1}$, Xiao W. Zhou ${ }^{2}$, Sergio F. Almeida ${ }^{1}$, Rodolfo Aguirre ${ }^{1} \&$ David Zubia $^{1}$ \\ ${ }^{1}$ Department of Electrical and Computer Engineering, the University of Texas at El Paso, Texas 79968, USA \\ ${ }^{2}$ Mechanics of Materials Department, Sandia National Laboratories, Livermore, California 94550, USA \\ Correspondence: Jose J. Chavez, Department of Electrical and Computer Engineering, University of Texas at El \\ Paso, TX 79968 USA. Tel: 1-650-273-6461. Email: jjchavez5@miners.utep.edu
}

$\begin{array}{lr}\text { Received: March 4, } 2016 \quad \text { Accepted: March 17, } 2016 \quad \text { Online Published: April 7, } 2016 \\ \text { doi:10.5539/jmsr.v5n3p1 } & \text { URL: http://dx.doi.org/10.5539/jmsr.v5n3p1 }\end{array}$

\begin{abstract}
Molecular dynamics simulations were used to catalogue atomic scale structures of CdTe films grown on eight wurtzite (wz) and zinc-blende (zb) CdS surfaces. Polytypism, grain boundaries, dislocations and other film defects were detected. Dislocation lines were distributed in three distinct ways. For the growths on the wz $\{0001\}$ and $\mathrm{zb}$ $\{111\}$ surfaces, dislocations were found throughout the epilayers and formed a network at the interface. The dislocations within the films grown on the wz $\{\overline{1} 100\}, \mathrm{wz}\{11 \overline{2} 0\}, \mathrm{zb}\{\overline{1} 10\}, \mathrm{zb}\{010\}$, and $\mathrm{zb}\left\{\frac{1}{10} 1 \frac{1}{10}\right\}$ surfaces formed an interface network and also threaded from the interface towards the film's surface. In contrast, the growth on the $\mathrm{zb}\{11 \overline{2}\}$ surface only had dislocations localized to the interface. This film exhibited a different orientation from the substrate to reduce the lattice mismatch strain energies, and therefore, its misfit dislocation density. Our study indicates that the substrate orientation could be utilized to modify the morphology of dislocation networks in lattice mismatched multi-layered systems.
\end{abstract}

Keywords: defects, dislocations, CdTe, substrates, surfaces, cadmium compounds, thin films

\section{Introduction}

Cadmium telluride (CdTe) thin films are widely used in photovoltaic (Colegrove et al., 2012) (McCandless and Sites 2003) (Okamoto, Yamada, \& Konagai 2001) and other optoelectronic applications (Liang et al., 2012) (Brill et al., 2005) (Heiss et al., 2006) due to its low production cost, a desired direct band gap of $\sim 1.5 \mathrm{eV}$, and an excellent absorption coefficient of $6 \times 10^{4} \mathrm{~m}^{-1}$ at $600 \mathrm{~nm}$. A variety of methods have been applied to synthesize CdTe films such as molecular beam epitaxy (MBE) (Kim et al., 2004) (Han, Kang, \& Kim 1999), physical vapour deposition (PVD) (Moutinho et al., 2008), high vacuum evaporation (HVE) (Terheggen et al., 2003), and close space sublimation (CSS) (Paudel, Xiao, \& Yan 2014) (Yan, Al-Jassim and Jones 2001). These efforts indicated that due to a large lattice mismatch with the underlying substrate (e.g., CdS), CdTe films always contain a high density of performance limiting lattice defects (Moseley et al., 2014), including point defects, stacking faults, dislocations, and grain boundaries.

Past efforts (Aguirre et al., 2014) (Cruz-Campa et al., 2012) (Zubia et al., 2007) have focused on reducing defects during the fabrication of the epilayers, although the fundamental mechanisms of defect formation are not well understood. Experimental studies have shown that careful preparation of the substrate surface termination (Myers et al., 1983) and the orientation of the substrate (Smith et al., 2000) (Sarney \& Brill, 2004) (Terheggen et al., 2003) can greatly influence the microstructure and the quality of the heteroepitaxial film. However, the high-resolution characterization techniques used to obtain these results are destructive, expensive, and time consuming. For example, transmission electron microscopy (TEM) (Li et al., 2013) (Yan, Al-Jassim, \& Jones, 2001) is effective at revealing two-dimensional (2-D) microstructures with atomic resolution, but cannot provide three-dimensional (3-D) information that determines material properties and defect evolution. Moreover, atomic probe tomography (Kelley \& Miller, 2007) (Miller \& Forbes, 2009) provides rich 3-D compositional information, but is unable to resolve lattice and defect structure with atomic resolution. Alternatively, atomistic simulations such as molecular dynamics (MD) (Alder \& Wainwright, 1959) (Rapaport, 2006) allow virtual experiments that isolate external factors and offer a 3-D analysis of the heteroepitaxial microstructures. More importantly, MD simulations can rapidly generate a large database of atomic scale structures of films as a function of substrate characteristics 
including all commonly encountered substrate orientations. A similar database may take many years to develop using experiments alone.

In recent years, the present authors have developed a robust MD method capable of simulating the growth of IIVI compounds (Zhou \& Ward, 2013). Based on this MD method, we have discovered and corrected issues of the traditional misfit dislocation theory, designed defect free, nanostructured CdTe/CdS solar cells (Zhou et al., 2015), and catalogued the atomic scale structures of CdS homoepitaxial films on commonly encountered CdS surfaces without the lattice mismatch effect (Almeida et al., 2016). In the present paper, we further extend the study to catalogue the atomic scale structures of heteroepitaxial CdTe films on commonly encountered $\mathrm{wz}$ and $\mathrm{zb} \mathrm{CdS}$ surfaces with the lattice mismatch effect. A separate study will also be performed to explore the mechanisms of the observed phenomena. Our ultimate objective is to create a convenient handbook characterizing substrate orientation effects on atomic scale structure of films that can help material scientists interpret experimental observations and reduce defects in multi-layered films via selection of substrate orientations.

\section{Molecular Dynamics Simulation Details}

The simulations were performed with the Large-Scale Atomic/Molecular Massively Parallel Simulator (LAMMPS) code (Plimpton, 1995) employing a Zn-Cd-Hg-S-Se-Te Stillinger-Weber potential (Zhou et al., 2013) capable of predicting the crystal structures and defects observed experimentally. The CdTe growth was performed on eight different wurtzite (wz) and zinc blende (zb) CdS substrate surfaces. The wz substrates included; $\{0001\},\{1100\}$, and $\{11 \overline{2} 0\}$ surfaces. The zb substrates included; $\{111\},\{11 \overline{2}\},\{\overline{1} 10\},\{010\}$, and $\left\{\frac{1}{10} 1 \frac{1}{10}\right\}$ surfaces. Here the $\left\{\frac{1}{10} 1 \frac{1}{10}\right\}$ surface essentially mimics a $\sim 8^{\circ}$ miscut from the $\{010\}$ surface. The CdS lattice constants were $\mathrm{a}_{\mathrm{wz}}=$ $4.134 \AA$ and $\mathrm{c}_{\mathrm{wz}}=6.752 \AA$ for the wz crystals, and $\mathrm{a}_{\mathrm{zb}}=5.847 \AA$ for the zb crystals. The lattice parameters for $\mathrm{wz}$ CdTe were $\mathrm{a}_{\mathrm{wz}}=4.590 \AA$ and $\mathrm{c}_{\mathrm{wz}}=7.495 \AA$, while the zb lattice parameter was $\mathrm{a}_{\mathrm{zb}}=6.491 \AA$. These lattice constant values were calculated from time averaged MD simulations of bulk single crystals at $300 \mathrm{~K}$ and are in excellent agreement (less than 1\% difference) with experimental values (Donnay and Ondik 1973) and molecular statics calculations (Zhou et al., 2013).

The deposition parameters were selected to ensure crystalline growth based on previous results (Zhou, Johnson, \& Wadley, 1997) (Zhou \& Wadley, 2000) (Zhou \& Wadley, 2001) and a complete description of the vapour deposition simulations methods has been discussed previously (Zhou et al., 2012). Briefly, CdTe growth was simulated at a deposition temperature of $1200 \mathrm{~K}$ under isothermal conditions by employing the Nose-Hoover algorithm (Hoover, 1985). The total deposition time was $\sim 19.6 \mathrm{~ns}$ with a deposition rate of $\sim 0.48 \mathrm{~nm} / \mathrm{ns}$. Periodic boundary conditions were employed in the $\mathrm{x}$ - and $\mathrm{z}$ - directions while a free boundary condition was employed in the $\mathrm{y}$ - (growth) direction. The adatoms had an incident energy of $5.0 \mathrm{eV}$ and a stoichiometric vapour ratio of $\mathrm{Cd}: \mathrm{Te}$ $=1: 1$. During simulations, positions of atoms in the bottom two monolayers of the substrates were fixed to prevent crystal drift due to adatom momentum transfer. It should be noted that the heteroepitaxy growth mechanism differs from that of homoepitaxy due to additional effects from lattice mismatch and chemistry. These effects are taken into account by a high-fidelity interatomic potential. The Stillinger-Weber potential employed in this work ensures the lowest energy configuration of the equilibrium compounds in addition to capturing their lattice constants and cohesive energies.

Following MD simulations of growth, a three-step post-deposition treatment was used to reduce thermal noises and improve the clarity of the data. First, an MD annealing (without adding more adatoms) was performed for $\sim 8$ ns to relax the structures. The MD simulation was then continued for another $\sim 8$ ns where the temperature was linearly cooled down to $50 \mathrm{~K}$. Finally, a molecular statics energy minimization simulation was carried out to relax the structures at $0 \mathrm{~K}$.

The effect of system size was explored and larger systems (between $\sim 200 \AA$ and $\sim 600 \AA$ in the lateral dimensions) generally captured a better physical description of the material defects at the expense of an increased computing time. In contrast, simulations that are less computationally intensive $(<\sim 100 \AA$ per side) could capture only a few single dislocation segments in general. The heterostructure samples employed had a size of $\sim 215 \AA, \sim 120 \AA$, and $\sim 160 \AA$ respectively for the $\mathrm{x}, \mathrm{y}$, and $\mathrm{z}$ dimensions. This system size appropriately captured the dislocation networks studied in this work while consuming an appropriate amount of computing resources.

\section{Analysis of MD Data}

The Open Visualization Tool (Stukowski, 2010) (OVITO) version 2.5.1 was employed to analyse the MD data and to render the images used in this work. The common neighbour analysis algorithm for diamond structures provided by the tool was used for structural characterization of the simulated films. The dislocation extraction algorithm (DXA) (Stukowski \& Albe, 2010) (Stukowski, Bulatov, \& Arsenlis, 2012) was used for the identification of linear defects within the data. Two types of dislocation analyses were explored for this study: one 
assumes a perfect wz lattice and the other assumes a perfect $\mathrm{zb}$ lattice. The Burger's vectors assigned to the dislocation segments were given in Miller-Bravais indices for the wurtzite analysis and cubic Miller index format for the zinc blende analysis.

\section{Results}

4.1 Lattice Structure Percentage and Dislocation Density

(a) $\square$ zinc blende $\square$ wurtzite
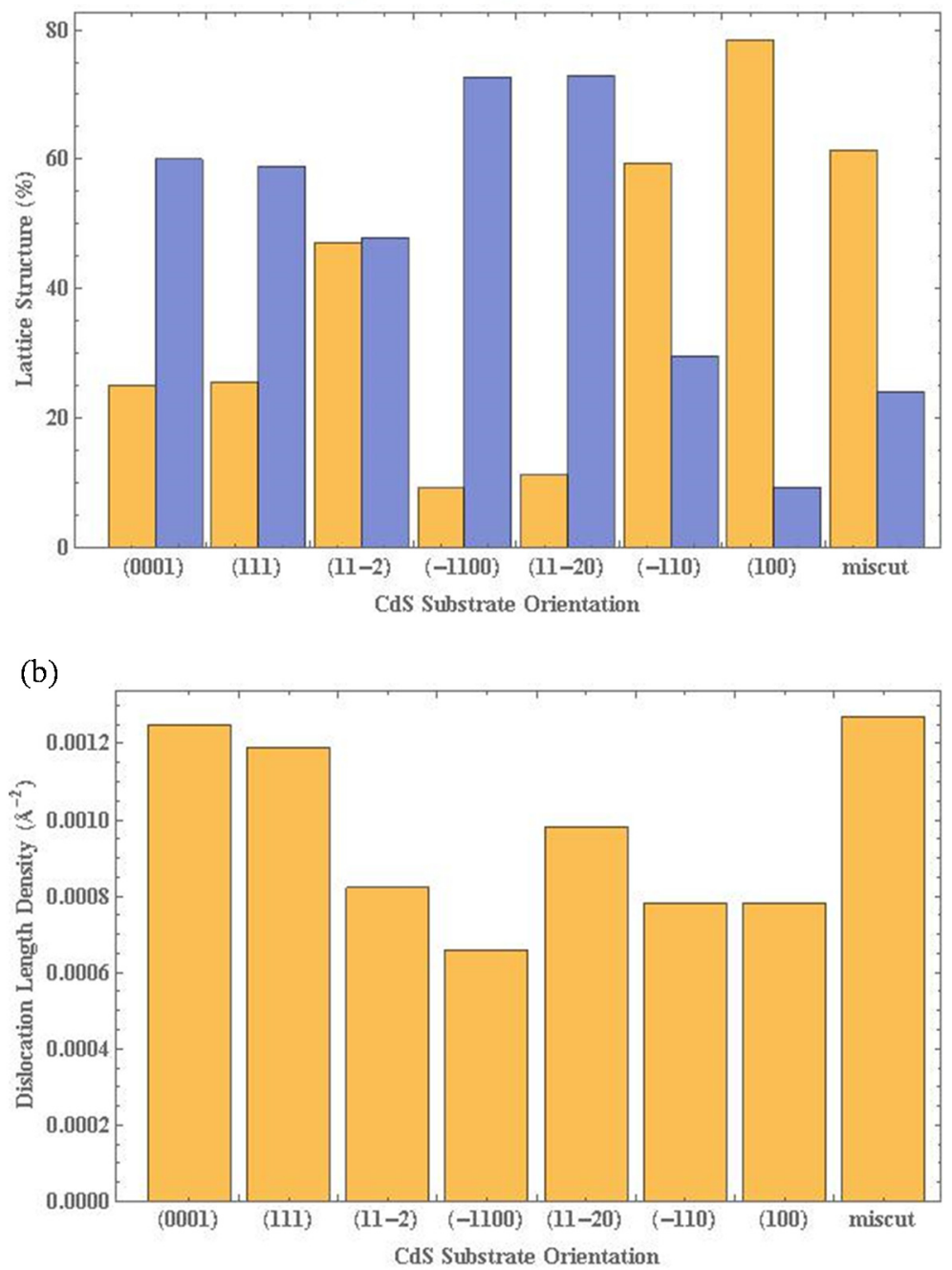

Figure 1. (a) Lattice structure percentage and (b) dislocation density for all simulated growths. Polytypism and misfit dislocations were observed in all cases

The lattice structure analyses results in Figure 1(a) show that each film contained multiple phase domains, having some amount of atoms associated to wurtzite and zinc blende structures. This polytypism is a common occurrence reported in CdTe films grown using MBE (Smith et al., 2000), as well as epitaxy using CSS (Al-Jassim et al., 2001) (Yan et al., 2000). Each simulated film also contained some regions where the structure did not match either the $w z$ or $z b$ lattice structures (unmatched percentages are not shown in the figure). These unmatched regions arise from other crystallographic imperfections such as point defects (vacancies, interstitials, etc.), grain boundaries, and dislocations. Visualizations of the atomic microstructure revealed that the epilayer grain orientation matched the substrate orientation in all cases except for the growth on the $\{11 \overline{2}\}$. The mechanism to form a new growth orientation will be reported in detail elsewhere. Film dislocation densities near the interface $(<\sim 100 \AA$ away from the substrate) were also analysed, and the results are shown in Figure 1(b) for all samples. The data indicates that films grown on the hexagonal surfaces $(\{0001\}$ and $\{111\})$ are highly defected. The remaining six surfaces have 
rectangular geometries. Except for the $\{11 \overline{2} 0\}$ and miscut surfaces, four of the growths on these surfaces clearly had lower dislocation densities when compared to hexagonal surface growths. The lowest dislocation density occurred for the $\{\overline{1} 100\}$ substrate. The trends observed in Figure 1 suggest that using rectangular rather than hexagonal substrate surfaces can reduce defect densities. The same trend was observed in MBE CdTe films grown on Si substrates (Terheggen et al., 2003). This was also observed in theoretical studies employing density functional theory (Yin et al., 2015) and in CdS homoepitaxy via MD (Almeida et al., 2016).

\subsection{Burgers Vector and Dislocation Spatial Distributions}

Analysis of the dislocation networks extracted using zinc blende DXA revealed three distinct spatial distribution categories associated with CdS substrate orientations; the $\{11 \overline{2}\}$ orientation, orientations with rectangular surface geometry $\left(\{\overline{1} 100\},\{11 \overline{2} 0\},\{\overline{1} 10\},\{010\},\left\{\frac{1}{10} 1 \frac{1}{10}\right\}\right)$, and orientations with hexagonal surface geometry $(\{0001\}$ and $\{111\})$. These three categories are represented in Figure 2 by the films grown on the $\{11 \overline{2}\},\{\overline{1} 100\}$, and $\{0001\}$ oriented substrates. All the dislocations occurred in the grown films and none were found in the CdS substrates.

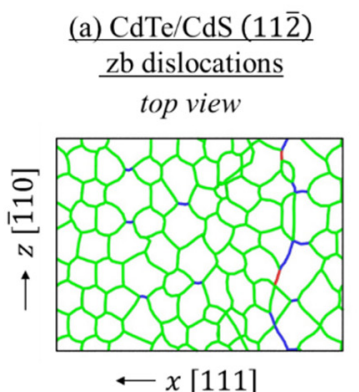

ross-section view

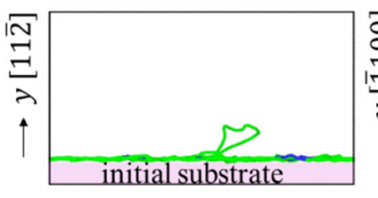

$\longleftarrow x[111]$ (b) $\mathrm{CdTe} / \mathrm{CdS}(\overline{1} 100)$ wz dislocations top view

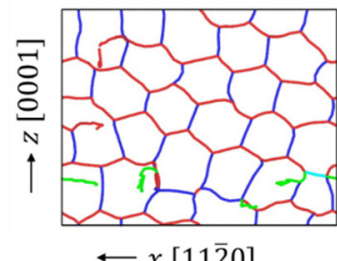

$x[1120]$

cross-section view

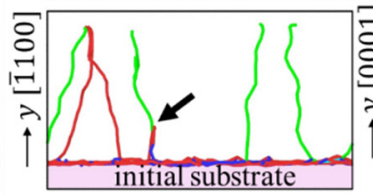

$\longleftarrow x[11 \overline{2} 0]$

Legend: $\quad \square 1 / 2<110>$ (full dislocation) $1 / 6<110>$ partial
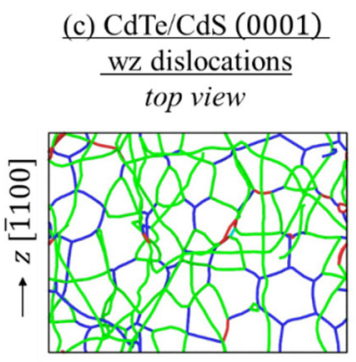

$\longleftarrow x[11 \overline{2} 0]$

cross-section view

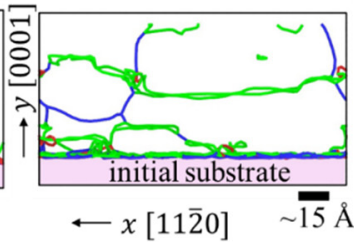

$\square 1 / 6<112>$ (Shockley partial)

Figure 2. Dislocation configurations in (a) CdTe on zb (11) $)$ CdS, (b) CdTe on wz (11100) CdS, and (c) CdTe on wz (0001) CdS substrate surfaces

The first distribution category consists of an interfacial dislocation network with virtually no dislocations in the bulk of the film as shown in Figure 2(a) suggesting a highly efficient mechanism at relieving lattice mismatch strain. The film grown on the $\{11 \overline{2}\}$ substrate was the only one that showed this type of dislocation distribution. The Burgers vector of the interfacial dislocations are predominantly $\frac{1}{6}<112>$ Shockley partials (or $\frac{1}{3}<1100>$ for a wz DXA). A small number of $\frac{1}{3}<112>$ dislocations were also detected and are shown in red colour in Figure 2(a).

The second dislocation category is shown in Figure 2(b), where in addition to a dislocation network at the interface, a group of dislocations threaded from the interface to the surface. This type of dislocation distribution was observed in the films grown on the $\{\overline{1} 100\},\{11 \overline{2} 0\},\{\overline{1} 10\},\{010\}$, and $\left\{\frac{1}{10} 1 \frac{1}{10}\right\}$ oriented CdS surfaces which possess a rectangular surface geometry. In this case, the Burgers vectors of the interfacial dislocations are roughly an equal mixture of $\frac{1}{2}<110>\left(\right.$ or $\left.\frac{1}{3}<1120>\right)$ full dislocations and $\frac{1}{6}<114>\left(\right.$ or $\left.\frac{1}{6}<2203>\right)$ shown in red colour in Figure 2(b). However, the threading dislocations in the cross-section view of Figure 2(b) are $\frac{1}{6}<112>$ Shockley partials and $\frac{1}{6}<114>$. These threading dislocations don't intersect and instead remain approximately parallel with respect to each other. In one instance (indicated by the black arrow), a vertical dislocation junction was formed between $\frac{1}{2}<110>$ and $\frac{1}{6}<114>$ dislocations near the interface, with one additional $\frac{1}{6}<112>$ segment threading towards the surface. 
The third dislocation category is shown in Figure 2(c), where dislocations were present throughout the film. This type of dislocation distribution was observed in the films grown on surfaces with hexagonal surface geometry, $\{0001\}$ and $\{111\}$. These films contained a mixture of $\frac{1}{6}<112>$ Shockley partial and $\frac{1}{2}<110>$ full dislocations both at the interface and in the bulk. The Burgers vectors of both $\frac{1}{6}<112>$ Shockley partials and $\frac{1}{2}<110>$ full dislocations lie on the closed-packed planes, and their formation is energetically favoured especially in materials with a low stacking fault energy that promote polytypism (Terheggen et al., 2003). Other types of dislocations were also detected but their densities were significantly lower.

\subsection{Lattice Structure Morphology}

The lattice structures of the CdTe films were further analysed. Figure 3 shows atomistic visualizations of the $\mathrm{CdTe} /\{11 \overline{2}\} \mathrm{CdS}$ heterostructure. Figure $3(\mathrm{a})$ is a cross-sectional view of a $\sim 10 \AA$ slice of the sample where the atoms are coloured according to species $\left(\mathrm{Cd}, \mathrm{S}\right.$, and Te). The ternary $\mathrm{CdS}_{\mathrm{y}} \mathrm{Te}_{1-\mathrm{y}}$ alloy was detected in a $10 \AA$ thick region straddling the interface. The $\mathrm{S}$ atoms were observed to diffuse further into the epilayer in comparison to the Te into the substrate, similarly to experimental observations using a TEM equipped with an energy dispersive Xray spectroscopy (EDS) detector (Terheggen et al., 2003) where low concentrations of S were detected to diffuse into the film but no Te diffusion into the substrate. This intermixing behaviour was a trend observed in all eight samples. Figure 3(b) is another cross-sectional view of the same data except that the atoms are now coloured according to their lattice structure. The light blue regions indicate a zinc blende structure and the red regions indicate a wurtzite structure. The orange atoms indicate atoms that could not be matched to either the $\mathrm{zb}$ or $\mathrm{wz}$ structures. This occurs in defected regions such as point defects (e.g., vacancies and interstitials), surfaces, grain boundaries, and dislocation cores. Comparison between Figures 3(a) and 3(b) clearly indicates the presence of polytypism in agreement with experimental reports (Smith et al., 2000) (Al-Jassim et al., 2001) (Yan et al., 2000) and stacking faults along a direction about $\sim 22^{\circ}$ from the interface plane. The majority of the grains have a zinc blende crystal structure.

Figure 3(c) shows a plan view lattice structure map near the interface as indicated by marker "C" in Figure 3(b). The orange atoms outline an interfacial dislocation network consistent with the dislocation network observed in Figure 2(a). In this region the film consisted of numerous grains with an average size of $\sim 15 \AA$. Figure $3(\mathrm{~d})$ shows a plan view lattice structure map about $80 \AA$ away from the interface (marked as "B" in Figure 3(b)). In contrast to the region near the interface, the film in Figure 3(d) consisted of a single grain with point defects and alternating $\mathrm{zb}$ and $\mathrm{wz}$ regions lying along the $\mathrm{x}$ direction.
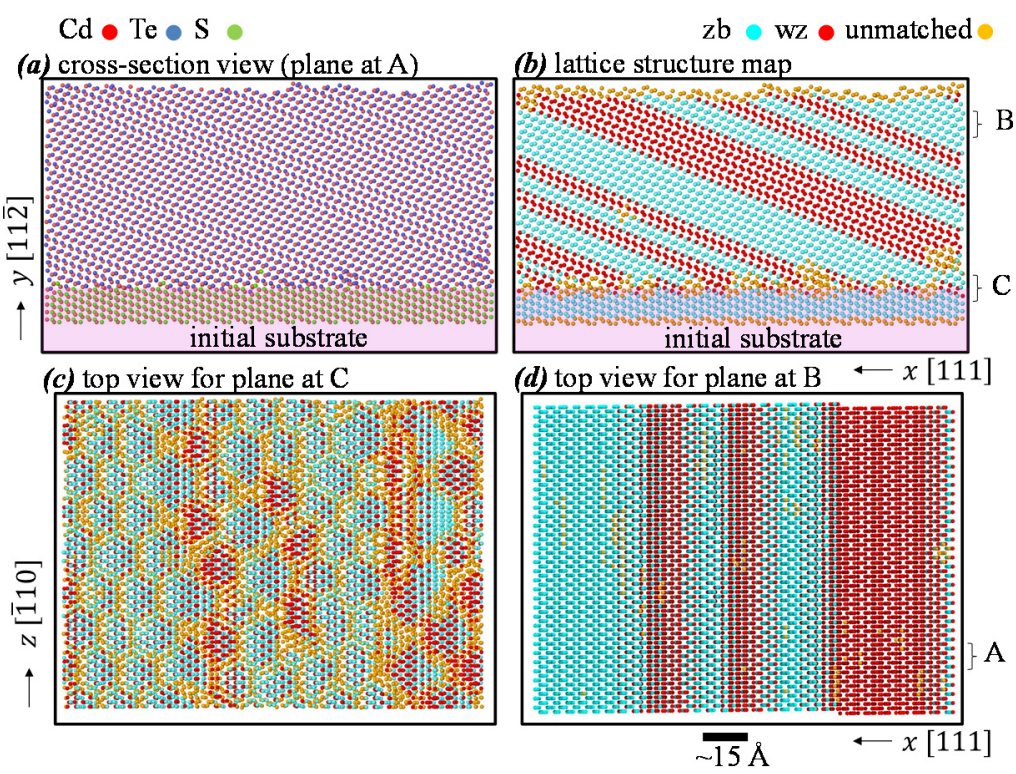

Figure 3. Visualization of the CdTe on $\mathrm{zb}(11 \overline{2}) \mathrm{CdS}$ : (a) front view where atoms are distinguished by species, (b) front view where atoms are distinguished by local structures as zinc blende (light blue), wurtzite (red), and "unmatched" (orange), (c) plan view of atom structures at a location near the interface, showing an average grain size of $\sim 15 \AA$ with zinc blende being the preferred structure, and (d) plan view of atom structures at a location

$\sim 80 \AA$ away from the interface, showing polytypism without grain boundaries other than point defects 
A similar analysis was performed for the $\mathrm{CdTe} /\{\overline{1} 100\} \mathrm{CdS}$ heterostructure in Figure 4. Figures 4(a) and 4(b) clearly indicate three different regions separated, for example, by the orange atoms in Figure 4(b). In this case, the orange atoms represent dislocation line cores which begin at the interface and extend to the surface. Point defects were detected within the grains (black arrow in Figure 4(b)). Figure 4(c) shows the dislocation network near the interface, in which the line defects connect to form geometries with an area of $\sim 18 \AA$. This is similar to the phenomenon observed for the $\{11 \overline{2}\}$ growth in Figure 3. In contrast, Figure 4(d) indicates that at $\sim 80 \AA$ away from interface the film consisted of a single wurtzite grain with stacking faults, point defects, and the intersecting dislocation cores (indicated by the black arrows for two cases) from the threading dislocations.
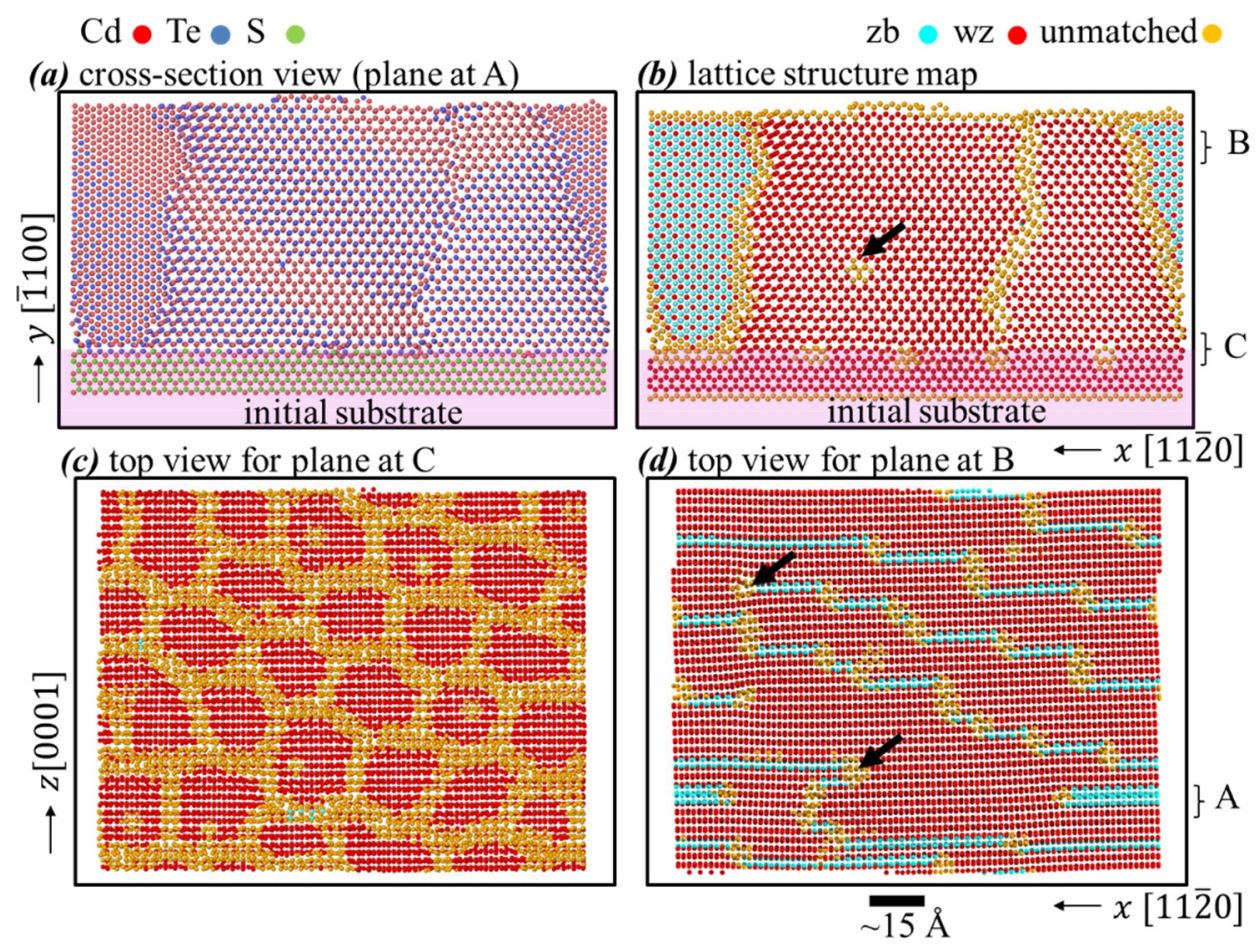

Figure 4. Visualization of the CdTe on wz (1100) CdS using the same approach described in Figure 3: (a) front view of the species map; (b) front view of the structure map; (c) plan view of the structure map near the interface, showing a dislocation network; and (d) plan view of the structure map at a location $\sim 80 \AA$ away from the interface, showing polytypism, point defects, and dislocation cores (indicated by black arrows)

The CdTe/ $\{0001\} \mathrm{CdS}$ heterostructure (Figures 5(a) and 5(b)) shows polytypism, stacking faults, point defects, and dislocation cores throughout the film, consistent with the dislocation distribution showed in Figure 2(c). A polycrystalline network with hexagonal geometry and equal mixture of $\mathrm{zb}$ and $\mathrm{wz}$ grains is present at the interface with an average grain size of $\sim 15 \AA$ as shown in Figure 5(c). This is anticipated since the CdS substrate has a hexagonal surface geometry and experimental growths on this surface geometry commonly report polytypism (Smith et al., 2000) (Al-Jassim et al., 2001) (Yan et al., 2000). However only $20 \AA$ away from the interface, the hexagonal geometry is replaced by much larger zb and wz grains of $\sim 80 \AA$ nominal size and no remnant of the underlying hexagonal geometry (Figure 5(d)). 

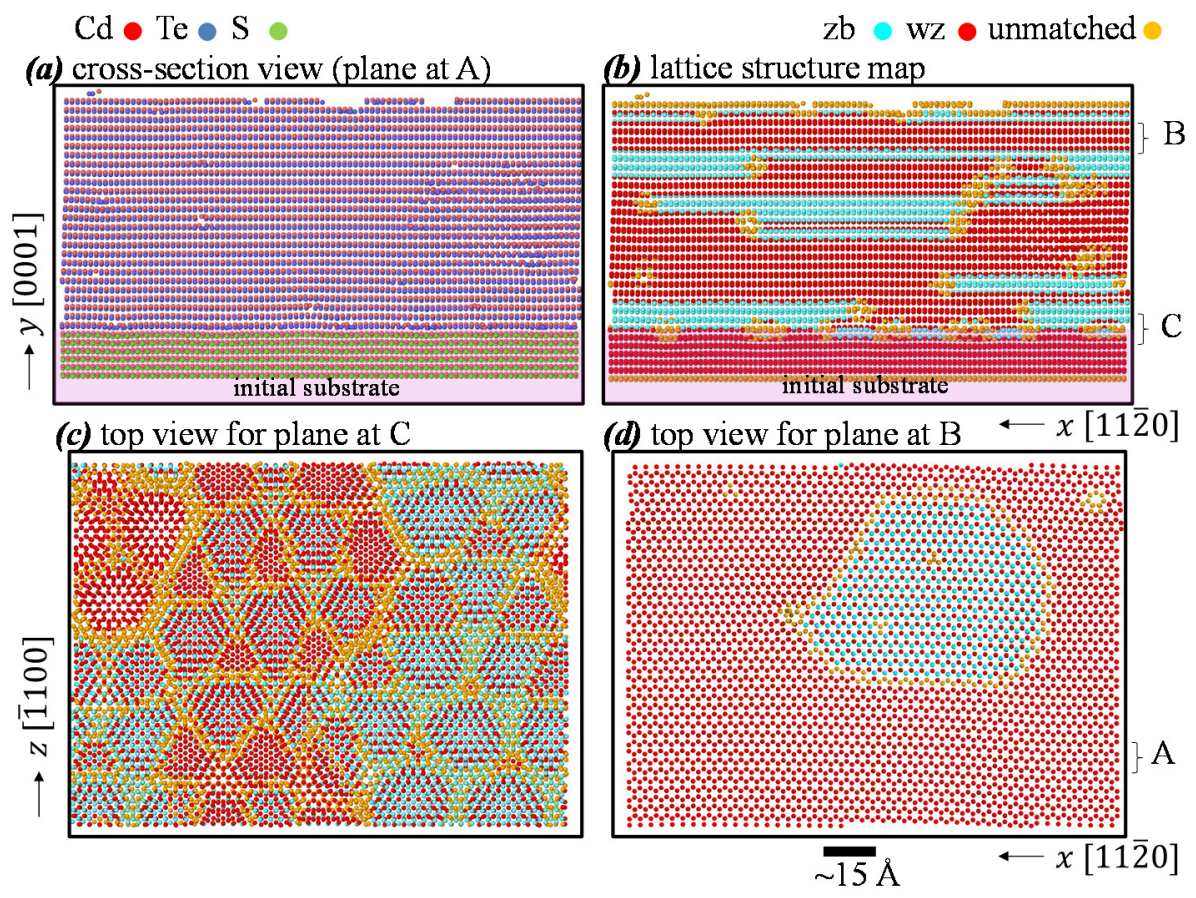

Figure 5. Visualization of the CdTe on wz (0001) CdS using the same approach described in Figure 3: (a) front view of the species map; (b) front view of the structure map showing polytypism, grain boundaries, and point defects; (c) plan view of the structure map near the interface, showing dislocation network, and an average grain size of $\sim 15 \AA$ with an even distribution of zinc blende and wurtzite grains; and (d) plan view of the structure map at a location $\sim 80 \AA$ away from the interface, showing polytypism in the form of a zinc blende grain surrounded by wurtzite material

\section{Conclusions}

Molecular dynamics simulations have been used to analyse CdTe films grown on eight commonly encountered CdS surfaces. The formation of the $\mathrm{CdS}_{\mathrm{y}} \mathrm{Te}_{1-\mathrm{y}}$ alloy was observed at the interface due to multi-layer intermixing in all growths. The lattice structure analysis detected polytypism (coexistence of wurtzite and zinc blende domains) in all samples. Most epilayers had a dominant lattice structure that matched that of its initial substrate, except for the growths on the $\{11 \overline{2}\}$ and $\{111\}$ surfaces. Lattice defects (point defects, stacking faults, dislocations and grain boundaries) were also found in all films. The film dislocation density was calculated and the data showed that growths on the hexagonal surfaces $(\{0001\}$ and $\{111\})$ are highly defected. In contrast, four of the growths on rectangular surfaces $(\{\overline{1} 100\},\{11 \overline{2}\},\{\overline{1} 10\}$, and $\{010\})$ had lower dislocation densities. The exception were the films grown on the $\{11 \overline{2} 0\}$ and miscut surfaces. These results agree with findings from a surface stability study (Yin et al., 2015) in which density functional theory was employed to explore optimal growth conditions. The dislocation morphology was found to depend on substrate orientation and could be described in three categories. For films grown on the $\{11 \overline{2}\}$ surface, dislocations were localized to the interface. An additional interesting phenomenon is that unlike other substrate surfaces, the film on the $\{11 \overline{2}\}$ surface grew in a new orientation different from the substrate. This accounts for the lack of misfit dislocations because the new orientation enables the lattice mismatch strain to be released. For films grown on the $\{\overline{1} 100\},\{11 \overline{2} 0\},\{\overline{1} 10\}$, $\{010\}$, and $\left\{\frac{1}{10} 1 \frac{1}{10}\right\}$ rectangular surfaces, dislocations formed at the interface but also threaded out towards the surface. For the films grown on the wz $\{0001\}$ and $\{111\}$ hexagonal surfaces, the dislocations formed a network at the interface and extended throughout the films. Our study indicates that selecting an appropriate substrate orientation could offer some control over defect density and dislocation network morphology that is formed within the epilayer.

\section{Acknowledgements}

Sandia National Laboratories is a multiprogram laboratory managed and operated by Sandia Corporation, a wholly owned subsidiary of Lockheed Martin Corporation, for the US Department of Energy's National Nuclear Security Administration under contract DE-AC04-94AL85000. This work was supported by DOE project No. EE0005958, 
National Science Foundation grants DGE-0903670 and CNS-1059430. This work used the Extreme Science and Engineering Discovery Environment (XSEDE), which is supported by NSF grant number ACI-1053575.

\section{References}

Aguirre, B. A., Zubia, D., Ordonez, R., Anwar, F., Prieto, H., Sanchez, C. A., ... \& Mcclure, J. C. (2014). Selective growth of CdTe on nano-patterned CdS via close-space sublimation. Journal of Electronic Materials, 43(7), 2651-2657. http://dx.doi.org/10.1007/s11664-014-3104-7

Alder, B. J., \& Wainwright, T. E. (1959). Studies in molecular dynamics. I. General method. The Journal of Chemical Physics, 31(2), 459-466. http://dx.doi.org/10.1063/1.1730376

Al-Jassim, M. M., Yan, Y., Moutinho, H. R., Romero, M. J., Dhere, R. D., \& Jones, K. M. (2001). TEM, AFM, and cathodoluminescence characterization of CdTe thin films. Thin Solid Films, 387(1), 246-250. http://dx.doi.org/10.1016/S0040-6090(00)01707-7

Almeida, S., Chavez, J. J., Zhou, X. W., \& Zubia, D. (2016). Effect of substrate orientation on CdS homoepitaxy by molecular dynamics. Journal of Crystal Growth, 441, 89-94. http://dx.doi.org/10.1016/j.jcrysgro.2016. 02.006

Brill, G., Chen, Y., Amirtharaj, P. M., Sarney, W., Chandler-Horowitz, D., \& Dhar, N. K. (2005). Molecular beam epitaxial growth and characterization of Cd-based II-VI wide-bandgap compounds on Si substrates. Journal of electronic materials, 34(5), 655-661. http://dx.doi.org/10.1007/s11664-005-0080-y

Colegrove, E., Banai, R., Blissett, C., Buurma, C., Ellsworth, J., Morley, M., ... \& Scott, M. (2012). High-efficiency polycrystalline $\mathrm{CdS} / \mathrm{CdTe}$ solar cells on buffered commercial TCO-coated glass. Journal of electronic Materials, 41(10), 2833-2837. http://dx.doi.org/10.1007/s11664-012-2100-z

Cruz-Campa, J. L., Zubia, D., Zhou, X., Aguirre, B. A., Ward, D., Sanchez, C. A., ... \& Lu, P. (2012, June). Nanopatterning and bandgap grading to reduce defects in CdTe solar cells. In Photovoltaic Specialists Conference (PVSC), 2012 38th IEEE (pp. 000838-000842). IEEE. http://dx.doi.org/10.1109/PVSC. 2012.6317734

Donnay, J, D. H., \& Ondik, H. M. (1973). Crystal Data Determinative Tables (3rd ed., Vol. 2, inorganic compounds). U.S.A. : U. S. Department of Commerce, National Bureau of Standards, and Joint Committee on Power Diffraction Standards.

Han, M. S., Kang, T. W., \& Kim, T. W. (1999). The dependence of the structural and optical properties on the CdTe epitaxial layer thicknesses in CdTe (111)/GaAs (100) heterostructures. Applied surface science, 140(1), 1-11. http://dx.doi.org/10.1016/S0169-4332(98)00363-8

Heiss, W., Groiss, H., Kaufmann, E., Hesser, G., Böberl, M., Springholz, G., ... \& Yano, M. (2006). Centrosymmetric $\mathrm{PbTe} / \mathrm{CdTe}$ quantum dots coherently embedded by epitaxial precipitation. Applied physics letters, 88(19), 192109. http://dx.doi.org/10.1063/1.2202107

Hoover, W. G. (1985). Canonical dynamics: equilibrium phase-space distributions. Physical Review A, 31(3), 1695. http://dx.doi.org/10.1103/PhysRevA.31.1695

Kelley, T F, \& Michael, K. M. (2007). Invited review article: atom probe tomography. Review of Scientific Instruments, 78, 031101. http://dx.doi.org/10.1063/1.2709758.

Kim, T. W., Lee, H. S., Lee, J. Y., Ryu, Y. S., \& Kang, T. W. (2004). Existence and atomic arrangement of microtwins in CdTe epilayers grown on GaAs (211) B substrates. Solid state communications, 129(8), 515518. http://dx.doi.org/10.1016/j.ssc.2003.11.047

Li, C., Poplawsky, J., Wu, Y., Lupini, A. R., Mouti, A., Leonard, D. N., ... \& Yan, Y. (2013). From atomic structure to photovoltaic properties in CdTe solar cells. Ultramicroscopy, 134, 113-125. http://dx.doi.org/10.1016/j.ultramic.2013.06.010

Liang, R., Xu, S., Yan, D., Shi, W., Tian, R., Yan, H., ... \& Duan, X. (2012). CdTe Quantum Dots/Layered Double Hydroxide Ultrathin Films with Multicolor Light Emission via Layer - by - Layer Assembly. Advanced Functional Materials, 22(23), 4940-4948. http://dx.doi.org/10.1002/adfm.201201367

McCandless, B. E., \& James, R. S. (2003). Cadmium Telluride Solar Cells. In Handbook of Photovoltaic Science and Engineering (pp. 617-662). John Wiley \& Sons, Ltd.

Miller, M. K., \& Forbes. R. G. (2009). Atom probe tomography. Materials Characterization, 60(6), 461-469. http://dx.doi.org/10.1016/j.matchar.2009.02.007 
Moseley, J., Al-Jassim, M. M., Kuciauskas, D., Moutinho, H. R., Paudel, N., Guthrey, H. L., ... Ahrenkiel, R. K. (2014). Cathodoluminescence analysis of grain boundaries and grain interiors in thin-film CdTe. Photovoltaics, IEEE Journal of, 4(6), 1671-1679. http://dx.doi.org/10.1109/JPHOTOV.2014.2359732

Moutinho, H. R., Dhere, R. G., Romero, M. J., Jiang, C. S., To, B., \& Al-Jassim, M. M. (2008). Electron backscatter diffraction of CdTe thin films: Effects of $\mathrm{CdCl} 2$ treatment. Journal of Vacuum Science \& Technology A, 26(4), 1068-1073. http://dx.doi.org/10.1116/1.2841523

Myers, T. H., Schetzina, J. F., Magee, T. J., \& Ormond, R. D. (1983). Growth of low dislocation density CdTe films on hydroplaned CdTe substrates by molecular beam epitaxy. Journal of Vacuum Science \& Technology A, 1(3), 1598-1603. http://dx.doi.org/10.1116/1.572275

Okamoto, T., Yamada, A., \& Konagai, M. (2001). Optical and electrical characterizations of highly efficient CdTe thin film solar cells. Thin Solid Films, 387(1), 6-10. http://dx.doi.org/10.1016/S0040-6090(00)01725-9

Paudel, N. R., Xiao, C., \& Yan, Y. (2014). Close-space sublimation grown CdS window layers for CdS/CdTe thinfilm solar cells. Journal of Materials Science: Materials in Electronics, 25(4), 1991-1998. http://dx.doi.org/10.1007/s10854-014-1834-1

Plimpton, S. (1995). Fast parallel algorithms for short-range molecular dynamics. Journal of computational physics, 117(1), 1-19. http://dx.doi.org/10.1006/jcph.1995.1039

Rapaport, D C. (2006). The Art of Molecular Dynamics. Cambridge: Cambridge University Press.

Sarney, W. L., \& Brill, G. (2004). A microstructural study of the CdTe/ZnTe film morphology as related to the Si substrate orientation. Solid-State Electronics, 48(10), 1917-1920. http://dx.doi.org/10.1016/j.sse.2004.05.036

Smith, D. J., Tsen, S. C., Chandrasekhar, D., Crozier, P. A., Rujirawat, S., Brill, G., ... \& Sivananthan, S. (2000). Growth and characterization of $\mathrm{CdTe} / \mathrm{Si}$ heterostructures - effect of substrate orientation. Materials Science and Engineering: B, 77(1), 93-100. http://dx.doi.org/10.1016/S0921-5107(00)00480-3

Stukowski, A., \& Albe, K. (2010). Extracting dislocations and non-dislocation crystal defects from atomistic simulation data. Modelling and Simulation in Materials Science and Engineering, 18(8), 085001. http://dx.doi.org/10.1088/0965-0393/18/8/085001

Stukowski, A. (2009). Visualization and analysis of atomistic simulation data with OVITO-the Open Visualization Tool. Modelling and Simulation in Materials Science and Engineering, 18(1), 015012. http://dx.doi.org/10.1088/0965-0393/18/1/015012

Stukowski, A., Bulatov, V. V., \& Arsenlis, A. (2012). Automated identification and indexing of dislocations in crystal interfaces. Modelling and Simulation in Materials Science and Engineering, 20(8), 085007. http://dx.doi.org/10.1088/0965-0393/20/8/085007

Terheggen, M., Heinrich, H., Kostorz, G., Romeo, A., Baetzner, D., Tiwari, A. N., ... \& Romeo, N. (2003). Structural and chemical interface characterization of CdTe solar cells by transmission electron microscopy. Thin Solid Films, 431, 262-266. http://dx.doi.org/10.1016/S0040-6090(03)00268-2

Yan, Y., Al-Jassim, M. M., \& Jones, K. M. (2001). Characterization of extended defects in polycrystalline CdTe thin films grown by close-spaced sublimation. Thin Solid Films, 389(1), 75-77. http://dx.doi.org/10.1016/S0040-6090(01)00841-0

Yan, Y., Al-Jassim, M. M., Jones, K. M., Wei, S. H., \& Zhang, S. B. (2000). Observation and first-principles calculation of buried wurtzite phases in zinc-blende CdTe thin films. Applied Physics Letters, 77(10), 14611463. http://dx.doi.org/10.1063/1.1308062

Yin, W. J., Yang, J. H., Zaunbrecher, K., Gessert, T., Barnes, T., Yan, Y., \& Wei, S. H. (2015). Surface stability and the selection rules of substrate orientation for optimal growth of epitaxial II-VI semiconductors. Applied Physics Letters, 107(14), 141607. http://dx.doi.org/10.1063/1.4932374

Zhou, X. W., \& Wadley, H. N. G. (2000). Atomistic simulation of the vapor deposition of Ni/Cu/Ni multilayers: Incident adatom angle effects. Journal of Applied Physics, 87(1), 553-563. http://dx.doi.org/10.1063/ 1.371899

Zhou, X. W., \& Wadley, H. N. G. (2001). The low energy ion assisted control of interfacial structure: ion incident angle effects. Surface science, 487(1), 159-170. http://dx.doi.org/10.1016/S0039-6028(01)01088-3 
Zhou, X. W., Ward, D. K., Martin, J. E., van Swol, F. B., Cruz-Campa, J. L., \& Zubia, D. (2013). Stillinger-Weber potential for the II-VI elements Zn-Cd-Hg-S-Se-Te. Physical Review B, 88(8), 085309. http://dx.doi.org/10.1103/PhysRevB.88.085309

Zhou, X. W., Johnson, R. A., \& Wadley, H. N. G. (1997). A molecular dynamics study of nickel vapor deposition: temperature, incident angle, and adatom energy effects. Acta materialia, 45(4), 1513-1524. http://dx.doi.org/10.1016/S1359-6454(96)00283-2

Zhou, X. W., Ward, D. K., Wong, B. M., Doty, F. P., Zimmerman, J. A., Nielson, G. N., ... \& Zubia, D. (2012). High-fidelity simulations of CdTe vapor deposition from a bond-order potential-based molecular dynamics method. Physical Review B, 85(24), 245302. http://dx.doi.org/10.1103/PhysRevB.85.245302

Zhou, X. W., Ward, D. K., Doty, F. P., Zimmerman, J. A., Wong, B. M., Cruz - Campa, J. L., ... \& McClure, J. C. (2015). A prediction of dislocation - free CdTe/CdS photovoltaic multilayers via nano - patterning and composition grading. Progress in Photovoltaics: Research and Applications, 23(12), 1837-1846. http://dx.doi.org/10.1002/pip.2628

Zubia, D., López, C., Rodríguez, M., Escobedo, A., Oyer, S., Romo, L., ... \& McClure, J. (2007). Ordered $\mathrm{CdTe} / \mathrm{CdS}$ arrays for high-performance solar cells. Journal of Electronic Materials, 36(12), 1599-1603. http://dx.doi.org/10.1007/s11664-007-0276-4

\section{Copyrights}

Copyright for this article is retained by the author(s), with first publication rights granted to the journal.

This is an open-access article distributed under the terms and conditions of the Creative Commons Attribution license (http://creativecommons.org/licenses/by/3.0/). 